\title{
Probability densities of superconductors
}

\author{
K. A. I. L. Wijewardena Gamalath*, G. D. K. N. Udeni \\ Department of Physics, University of Colombo, Colombo 3, Sri Lanka \\ *E-mail address: imalie@phys.cmb.ac.lk
}

\begin{abstract}
The energy, current density and momentum probability densities of superconductors were studied from London, Ginzburg-Landau and BSC theories by treating cooper pair as a particle moving in a magnetic field through analytical and numerical techniques. The London and GL solution were exactly the same at the classical limit for $N b N$. Considering a Cooper pair as a complete classical particle, the momentum probability density was derived by using the Maxwell velocity distribution and the quantum mechanical momentum probability density was derived by using the radial wave function of the cooper pairs for $\mathrm{Zn}$. The quantum mechanical and classical momentum probability densities overlap at zero momentum.
\end{abstract}

Keyword: Superconductors; Cooper pair; London theory; Ginzburg-Landau theory; BSC theory; current densities; momentum probability densities

\section{INTRODUCTION}

The discovery that the electrical resistance of various metals disappeared below a critical temperature $T_{c}$ by Onnes [1] in 1911 led to the finding of perfect diamagnetism in superconductors by Meissner and Ochsenfeld in 1933 [2]. A simple phenomenological theory for the electrodynamics properties of superconducting phenomena, that is the vanishing static electrical resistivity and an expulsion of the magnetic field from the interior was proposed by London brothers [3], relating current to electromagnetic fields in and around a superconductor. In GinzburgLandau theory [4], a mathematical theory proposed in predicting that free energy of a superconductor near the superconducting transition, expressed in terms of a complex order parameter field by minimizing the free energy with respect to fluctuations in the order parameter and the vector potential, the Ginzburg-Landau equations were obtained by predicting two new characteristic lengths, coherence length and penetration depth.

They observed the existence of two types of superconductors type I and type II, depending on the energy of the interface between the normal and superconducting states. Although coherent states can be defined for fermions as well as bosons, as single fermion coherent states are not directly useful in the theory of superconductivity they must be defined for fermion pairs. The origin of electron pairing during a superconducting phase was studied by Cooper [5] calculating the bound states of electrons having a lower energy than the Fermi energy subject to a small attractive force in a metal. In conventional superconductors, this attraction is due to exchange of virtual phonons which does not confirm to the boson communication rule. A cooper pair which is quite massive by molecular standards has orthogonal and correlate wave functions. In 1957 Bardeen Cooper and Schrieffer described superconductivity as a microscopic effect caused by a condensation of Cooper pairs into a boson-like state in BCS theory of superconductivity [6]. BCS state and the GinzburgLandau theory provides a direct connection to the order parameter of the coherent states of the electron pairs [7]. 
In the present paper, the superconductors were studied by obtaining the equations from the energy basis, current density basis and the momentum probability density basis of the cooper pairs. Classically, cooper pairs were assumed to behave like a real gas, and the Maxwell-velocity distribution was used to obtain the momentum probability density. The quantum mechanical momentum probability density was obtained by using the Hamiltonian of the cooper pairs. Graphical interpretations of the analytical and numerical solutions for the current density states of $\mathrm{NbN}$ and momentum probability densities of $\mathrm{Zn}$ are presented.

\section{CURENT DENSITY IN LONDON THEORY}

A superconductor, a material that losses all its resistivity, is a perfect conductor, that is to say, it is a material with infinite conductivity. As the current is finite, from Ohms law, the electric field will be zero and by Maxwell equations, the magnetic induction $\boldsymbol{B}$ will be a constant in time indicating that superconducting state is not an equilibrium state but a metastable state. Below a certain critical temperature, $T_{c}\left(\approx m^{-1 / 2}\right)$, which changes only when the mass of the ion in the lattice is changed while the rest remains

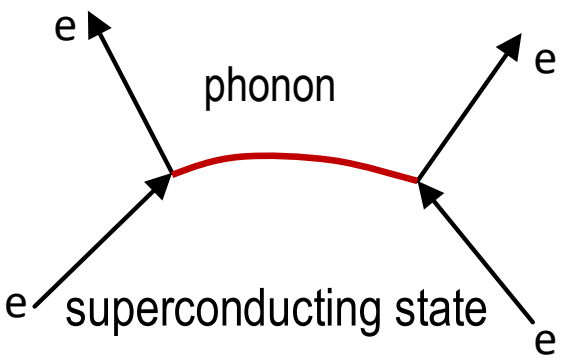

Figure 1. Cooperpair. exactly the same, a phase transition occurs to the electrons that are allowed to travel through the space formed by the regular arrangement of ions. In this new phase, called the superconducting state, they arrange themselves into Cooper pairs (Figure 1) where the correlation due to the polarization induced by one electron, constitute an attraction potential to a second electron. A Cooper pair is a bound state of two electrons, with energy at the Fermi surface having spins and vector momentum of opposite sign held together by interchange of a phonon between two electrons through the lattice. The interactions make it explicit so that no current is kept by the lattice and therefore no resistivity can manifest. Cooper pairs behave like a gas of electrons, a Fermi gas, each particle having twice the mass of electron $\left(m_{e}\right)$ and twice the electron charge $(-e)$ moving in the presence of a magnetic field. By assuming that the cooper pair electrons interact attractively with each other but do not interact with the each other electrons except via Pauli exclusion principle, the classical Hamiltonian representing the energy of the system can be written in terms of generalized momentum $p_{i}$ vector potential $\boldsymbol{A}(\boldsymbol{r}, t)$ and attractive potential $V(\boldsymbol{r})$ of the cooper pairs,

$$
H_{c l}=\frac{1}{2 m} \sum_{i=1}^{n_{s}}\left(p_{i}-q A_{i}(\boldsymbol{r}, t)\right)^{2}+q V(\boldsymbol{r})
$$

where $m=2 m_{e}, q=-2 e$ and $n_{s}$ is the density of superconducting carriers. The attractive potential between two such electrons that extends over the distance $l$ is assumed to be a constant such that

$$
\begin{aligned}
V(\boldsymbol{r}) & =-V_{0}=-\frac{e^{2}}{4 \pi \varepsilon_{0} d^{2}} \delta \quad r \leq l \\
& =0 \quad r \geq l
\end{aligned}
$$


where $d$ is the distance between the two electrons and $\delta$ is the displacement. The attractive potential is very small. Therefore for distance greater than $l$, the attractive potential $V(\boldsymbol{r}) \approx 0$, and the classical energy of the system become

$$
E_{c l}=\frac{1}{2 m} \sum_{i=1}^{n_{s}}\left(p_{i}-q A_{i}(\boldsymbol{r}, t)\right)^{2}=\frac{n_{s}}{2 m}(p-q A)^{2} .
$$

Since in quantum mechanics, operators represent observable quantities, the quantum mechanical Hamiltonian of a charged particle moving in a magnetic field read

$$
\hat{H}=\frac{1}{2 m}\left(\hat{p}_{i}-q \hat{A}_{i}(\boldsymbol{r}, t)\right)^{2}+q \hat{V}(\boldsymbol{r})
$$

Ginzburg and Landau [4] assumed that in a superconductor, the superconducting electrons, that is the superfluid part is described by a single one-particle wave function $\psi(\boldsymbol{r})$. They imposed the plausible normalization, that it vanishes in the normal state, but take some finite value below the critical temperature usually normalized to the density of superconducting carriers $n_{s}$. One-particle wave function is given by

$|\psi(\boldsymbol{r})\rangle=\sqrt{n}_{s}(\boldsymbol{r}) e^{i(\boldsymbol{k} \cdot \boldsymbol{r})}$

In order to find the energy eigenvalues, the time independent Schrodinger equation can be used:

$$
\hat{H}|\psi(\boldsymbol{r})\rangle=E|\psi(\boldsymbol{r})\rangle
$$

Substituting the Hamiltonian given in equations 4 and wave function given in equation 5 into time independent Schrodinger equation with

$\hat{p}_{i}=-i \hbar \nabla, \hat{A}_{i}(\boldsymbol{r}, t)=A(\boldsymbol{r}, t), \hat{V}(\boldsymbol{r})=V(\boldsymbol{r})=0$, the above equation read,

$$
\hat{H}|\psi(\boldsymbol{r})\rangle=\left\{\frac{1}{2 m}(-i \hbar \nabla-q A(\boldsymbol{r}, t))(-i \hbar \nabla-q \boldsymbol{A}(\boldsymbol{r}, t))\right\}|\psi(\boldsymbol{r})\rangle=\frac{1}{2 m}(\hbar \mathrm{k}-q A(\boldsymbol{r}, t))^{2}|\psi(\boldsymbol{r})\rangle=E|\psi(\boldsymbol{r})\rangle .
$$


The expected value of the energy is,

$$
\langle E\rangle=\langle\psi(\boldsymbol{r})|E| \psi(\boldsymbol{r})\rangle=E=\frac{1}{2 m}(\hbar \mathrm{k}-q A(\boldsymbol{r}, t))^{2}|\psi(\boldsymbol{r})|^{2}=\frac{n_{s}}{2 m}(p-q A(\boldsymbol{r}, t))^{2}
$$

As one electron in the Cooper pair has momentum $\hbar \boldsymbol{k}$ and the other $-\hbar \boldsymbol{k}$, when a superconductor is not carrying a current, the pair has zero net momentum with no net drift velocity. But when a current is flowing, there is a net drift velocity $v$ and a net momentum $m \boldsymbol{v}$ along the direction of electron flow. Since the coupling by virtual phonons takes place between electrons with momentum $\left(m_{e} \boldsymbol{v}+\hbar \boldsymbol{k}\right)$ and $\left(m_{e} \boldsymbol{v}-\hbar \boldsymbol{k}\right)$, the pair momentum is $m \boldsymbol{v}$. If a weak magnetic field is applied parallel to the surface of a superconductor, then the vector potential $\boldsymbol{A}$ in an appropriate gauge is parallel to the surface and if the nodal planes are also assumed to be parallel to the surface, then the drift momentum $\boldsymbol{p}$ is $2 m_{e} \boldsymbol{v}$. If the magnetic vector potential $\boldsymbol{A} \neq 0$, the pair is in motion with velocity $\boldsymbol{v}=\boldsymbol{e A} / m_{e}$ [8]. With de Broglie hypothesis $p=\hbar k$, the equation $8 \mathrm{read}$ :

$$
\langle E\rangle=\frac{4 n_{s} e^{2}}{m_{e}} A^{2}(\boldsymbol{r}, t) .
$$

The magnetic vector potential $\boldsymbol{A}$ can be obtained from magnetic induction $\boldsymbol{B}(\boldsymbol{\nabla} \times \boldsymbol{A})$. For a superconducting vortex with a cylindrical core of a radius $r$ and approximate coherence length $\lambda$, the resulting magnetic field of the vortex, along the cylindrical core taken to be in the $\mathrm{z}$-direction can be written in the form

$$
B_{z}(r)=\frac{\phi_{0}}{2 \pi \lambda^{2}} \ln \left(\frac{r}{\lambda}\right)
$$

where flux per vortex line $\phi_{0}=h / e$. The vector potential can be derived by using the London gauge $\nabla \cdot \boldsymbol{A}=0$. There is a minimum value of the total flux through any normal domain. The smallest domain is a quantized vortex or $u_{x}$ line, a tube-like region in which the order parameter and magnetic field take the values which would be excluded in the equilibrium bulk of the superconductor.The vector potential can be derived by using gauge invariant London gauge:

$$
A_{\phi}(r)=\frac{\phi_{0} r}{2 \pi \lambda^{2}} \ln \left(\frac{r}{\lambda}\right)
$$

Substituting the value of the vector potential in the expression given in equation 8 , the average energy:

$$
\langle E\rangle=\frac{4 n_{s} e^{2}}{m_{e}}\left(\frac{\phi_{0} r}{2 \pi \lambda^{2}} \ln \left(\frac{r}{\lambda}\right)\right)^{2}
$$


The London equation based on a two-fluid picture, a normal fluid and a super fluid gives the relation between the electric current density inside a superconductor [3]. The supercurrent is given by

$\boldsymbol{J}_{s}=-e n_{s} \boldsymbol{v}_{s} \quad$ and $\quad \frac{\partial \boldsymbol{J}_{s}}{\partial t}=-e n_{s} \frac{\partial \boldsymbol{v}_{s}}{\partial t}=\frac{e^{2} n_{s}}{m} \boldsymbol{E}$

Taking the curl of equation 13 with Maxwell's equation of Faraday's law of induction,

$\nabla \times \frac{\partial \boldsymbol{J}}{\partial t}=\frac{\partial}{\partial t}(\nabla \times \boldsymbol{J})=\frac{e^{2} n_{s}}{m}(\nabla \times \boldsymbol{E})=\frac{e^{2} n_{s}}{m}\left(-\frac{\partial \boldsymbol{B}}{\partial t}\right)$

Integrating equation 14 with the conditions that initially there is no supercurrent and magnetic field,

$\nabla \times \boldsymbol{J}=-\frac{e^{2} n_{s}}{m} \boldsymbol{B}$

From Maxwell's equation of Ampère's circuital law,

$(\nabla \times \boldsymbol{B})=\mu_{0} \boldsymbol{J}$

Taking the curl of equation 16 and substituting to equationin 15 :

$\nabla \times(\nabla \times \boldsymbol{B})=-\mu_{0} \frac{n_{s} e^{2}}{m_{e}} \boldsymbol{B}=-\frac{1}{\lambda^{2}} \boldsymbol{B}$

where $\lambda$ is the penetration depth, the distance inside the surface over which an external magnetic field is screened out to zero with the assumption that there is no magnetic field in the bulk of the superconductor. The London equation giving the relation between the electric current density inside a superconductor, to the magnetic vector potential $\boldsymbol{A}$ can be obtained from in equation 15,

$\nabla \times \boldsymbol{J}=-\frac{e^{2} n_{s}}{m}(\nabla \times \boldsymbol{A}) \Rightarrow \boldsymbol{J}_{L}=-\frac{n_{s} e^{2}}{m_{e}} \boldsymbol{A}=-\frac{1}{\mu_{0} \lambda^{2}} \boldsymbol{A}$.

This equation is evidently not gauge-invariant. Charge conservation requires $\boldsymbol{\nabla} \cdot \boldsymbol{J}=0$ and thus the vector potential must be transverse. $\boldsymbol{\nabla} \cdot \boldsymbol{A}=0$ is called the London gauge. Equation 18 can be used to find the simple mathematical description of a superconducting vortex having a cylindrical core of a radius $r$ of approximately the coherence length $\lambda$. The resulting magnetic field of the vortex, along the cylindrical core taken to be in the z-direction is given in equation 10 and the vector potential derived by using gauge invariant London gauge in equation 11. Substituting the value of vector potential in equation 18 , current density can be obtained as

$J_{L}=-\frac{n_{s} e^{2}}{m_{e}} \frac{\phi_{0}}{2 \pi \lambda^{2}} r \times \ln \left(\frac{r}{\lambda}\right)$ 
The current density for the superconducting metal, niobium nitrite $\mathrm{NbN}$ [4] against the radial distance, $r(\varepsilon \leq r \leq \lambda)$ between the coherence length $\varepsilon$ of the superconducting material in the range of $3-5 \mathrm{~nm}$ and for the penetration depth $\lambda$ in the range of $200-350 \mathrm{~nm}$ is shown in Figure 2.
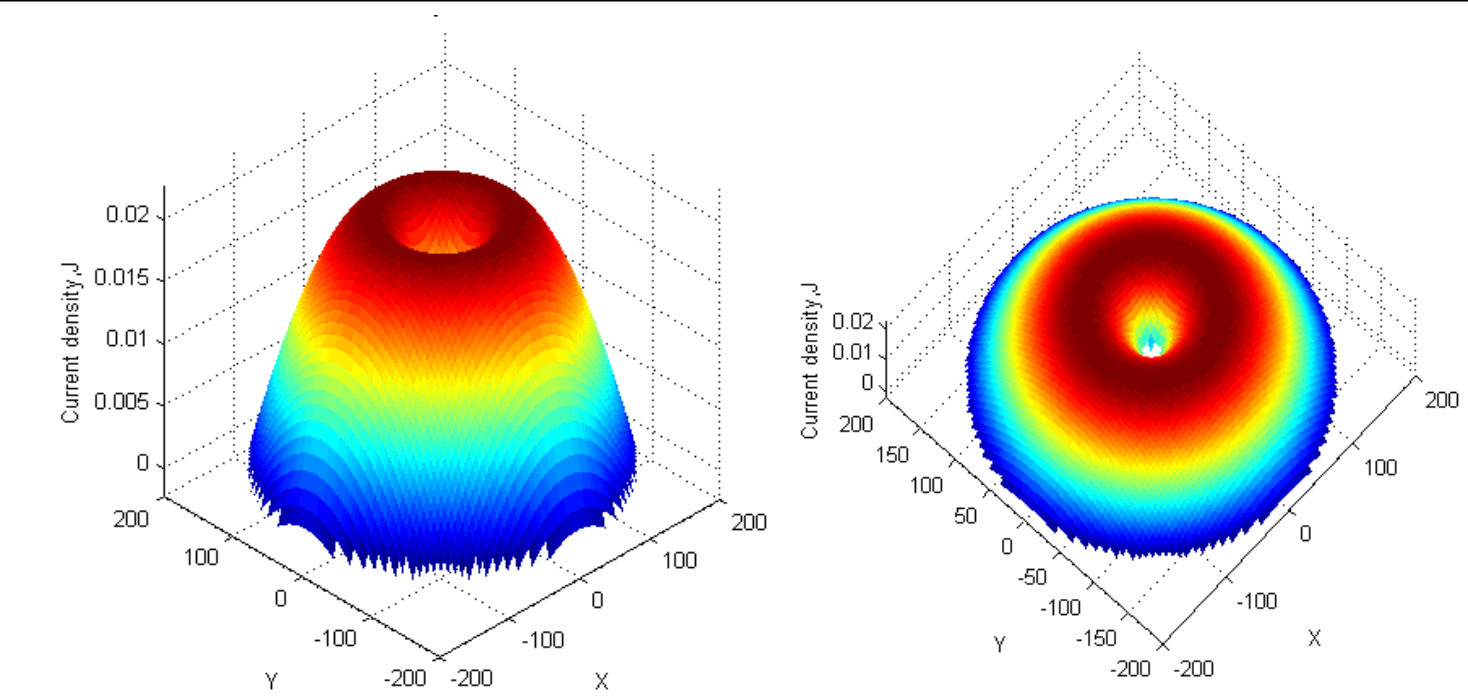

Figure 2. Side view (left) and top view (right) of the variation of the classical current density with core radius $r$ for $\mathrm{NbN}(\varepsilon=3-5 \mathrm{~nm}$ and $\lambda=200-350 \mathrm{~nm})$ in London theory.

\section{CURENT DENSITY IN GL THEORY}

The superconducting state has lower entropy than the normal state and therefore is the more ordered state. A general theory introduced by Landau, based on order parameter to describe phase transitions with just a few reasonable assumptions is remarkably powerful. In this context, an order parameter is a thermodynamic variable that is zero on one side of the transition and non-zero on the other. Based on an analogy with Bose-Einstein condensation, The thermodynamic free energy $F$ of the system is assumed to be an analytic function of density of superconducting carriers $n_{s}$, so that its value $F_{S}$ in the superconducting state can be expanded in a power series around its value in the normal state $F_{n}$ close to the critical temperature $T_{c}$ :

$F_{s}=F_{n}+a n_{s}+\frac{b}{2} n_{s}^{2}+\ldots$

In the conventional description, known as the Landau model, one assumes that sufficiently close to $T_{c}$ the free energy density relative to that of the normal state can be expanded in a Taylor series in the order parameter $\psi$ :

$f(\psi)=\alpha \psi^{2}+\frac{\beta}{2} \psi^{4} \quad(\beta>0)$

This assumes that the order parameter is real and that the free energy density is an even function of the order parameter. The phase transition takes place at $\alpha\left(T_{c}\right)=0$. Thus, a power series expansion of $\alpha(T)$ around $T_{\mathrm{c}}$ may be expected to have the following leading form:

$\alpha=a\left(T-T_{c}\right) \quad(a>0)$ 
In the Ginzburg-Landau (GL) theory, however, $\psi$ is assumed to be complex rather than real as is the case for a macroscopic wave function. Crucially, $\psi$ couples to the electromagnetic field in the same way as for an ordinary wave function. Then canonical momentum has to be replaced by the kinetic momentum:

$-i \hbar \nabla \rightarrow-i \hbar \nabla-q A$

where $\boldsymbol{A}$ is the vector potential and $q=-2 e$ is the relevant charge. A final part in the free energy is the relevant magnetic field energy density $B^{2} / 2 \mu_{0}$. Therefore the Ginzburg-Landau free energy density, the condensation energy:

$$
f_{G L}=f_{s}-f_{n}=\alpha|\psi|^{2}+\frac{\beta}{2}|\psi|^{4}+\frac{1}{2 m}|(-i \hbar \nabla+2 e A) \psi|^{2}+\frac{B^{2}}{2 \mu_{0}}
$$

where the effective mass $m=2 m_{e}$. To obtain the total free energy, the electromagnetic field energy must be included at each point $r$ and integrated over the system. Therefore the total free energy of the superconductor and the magnetic field is

$$
\mathrm{F}_{G L}(T)=\int\left(\frac{\hbar^{2}}{2 m}|(-i \hbar \nabla+2 e A) \psi|^{2}+\alpha|\psi|^{2}+\frac{\beta}{2}|\psi|^{4}\right) d^{3} r+\frac{1}{2 \mu_{0}} \int B^{2}(r) d^{3} r
$$

The first integral is carried out over the point $r$ inside the sample, while the second is performed over all space. Minimizing the free energy or condensation energy with respect to the wave function and the vector potential respectively leads to the celebrated Ginzburg-Landau equations [4]:

$-\frac{\hbar^{2}}{2 m}\left(\nabla+i \frac{2 e}{\hbar} A\right)^{2} \psi(\mathrm{r})+\left(\alpha+\beta|\psi|^{2}\right) \psi(r)=0$

A superconductor can be considered as a boson gas. By approximate an interacting ground state of a boson system by placing all bosons in the same state $\Psi(\boldsymbol{r})$ and using Bose version of Hartree-Fock theory, the $\Psi(\boldsymbol{r})$ is proportional to order parameter $\psi(\boldsymbol{r})$ and the GL free energy is the variational expectation. The term $|(-i \hbar \nabla+2 e A) \psi|^{2} / 2 m$ relates to the kinetic energy term and the $|\psi|^{4}$ term due to the interaction term. The dynamics of the macroscopic fields could be also derived from the microscopic hypothesis that all bosons have the same wave function $\Psi(\boldsymbol{r})$ and if the number of bosons is given by $N_{b}$, then order parameter $\psi(\boldsymbol{r})$

$\psi(\boldsymbol{r})=\sqrt{N_{b}} \Psi(\boldsymbol{r})$

The result is the time-dependent Ginzburg-Landau equation given in equation 25 . Using the order parameter and gauge invariant vector potential $\boldsymbol{\nabla} \cdot \boldsymbol{A}=0$ called the Landon gauge, in Ginzburg-Landau equation of the current density becomes

$$
\boldsymbol{J}=\frac{1}{\mu_{0}} \nabla \times \boldsymbol{B}=i \frac{q \hbar}{2 m}\left(\psi^{*} \nabla \psi-\psi \nabla \psi^{*}\right)-\frac{4 e^{2}}{m}|\psi|^{2} \boldsymbol{A}
$$


In the limit of uniform wave function or order parameter $\psi(\boldsymbol{r})$ equation 28 read

$$
\boldsymbol{J}=-\frac{4 e^{2}}{m}|\psi|^{2} \boldsymbol{A}
$$

The order parameter field, $\psi(\boldsymbol{r})$

$$
\psi(\boldsymbol{r}, t)=|\psi(\boldsymbol{r}, t)| e^{i \theta(\boldsymbol{r})}=\sqrt{n_{s}} e^{i \theta(\boldsymbol{r})}
$$

Here the arbitrary choice of $\theta$ expresses the spontaneous breaking of the continuous gauge symmetry, while the magnitude $|\psi|$ expresses the strength of superconductivity with $|\psi|=0$

$$
\boldsymbol{J}=-\frac{q}{m}(\hbar \theta+q A)|\psi|^{2}
$$

At the classical limit $\hbar \rightarrow 0$, the Ginzburg-Landau equation given in equation 31 becomes equation 29. If the magnetic vector potential $\boldsymbol{A} \neq 0$, the pair is in motion with velocity $\boldsymbol{v}=q \boldsymbol{A} / \mathrm{m}$ with de Broglie hypothesis $p=\hbar k$, equation 31 read:

$$
\boldsymbol{J}=-\frac{q}{m}(\boldsymbol{p}+q \boldsymbol{A})|\psi|^{2}=-\frac{3 q^{2} \boldsymbol{A}}{2 m}|\psi|^{2}
$$

Substituting the vector potential obtained for the London gauge in equation 11, for flux per vortex line, the equation 33 read

$$
\boldsymbol{J}=-\frac{3 q^{2} \phi_{0}}{2 m 2 \pi \lambda^{2}} \boldsymbol{r} \times \ln \left(\frac{\boldsymbol{r}}{\lambda}\right)|\psi|^{2}=-\frac{3 q^{2} n_{s} \phi_{0}}{4 m 2 \pi \lambda^{2}} \boldsymbol{r} \times \ln \left(\frac{\boldsymbol{r}}{\lambda}\right)=-\frac{3 e^{2} n_{s} \phi_{0}}{2 m_{e} 2 \pi \lambda^{2}} \boldsymbol{r} \times \ln \left(\frac{\boldsymbol{r}}{\lambda}\right)
$$

The GL current density as a function of position vector $r(\varepsilon \leq r \leq \lambda)$ for the superconducting metal, niobium nitrite $\mathrm{NbN}$ [4] between the coherence length $\varepsilon$ in the range of $3-5 \mathrm{~nm}$ and the depth $\lambda$ in the range of $200-350 \mathrm{~nm}$ is shown in Figure 3. The variations of the current densities in classical limit with respect to radius using London and GL method are shown in Figure 4. Brown colour corresponds to the London current density while the blue colour corresponds to GL current density. Since the cooper pair has a small velocity which results a tiny momentum, the difference between the results do not vary much. 

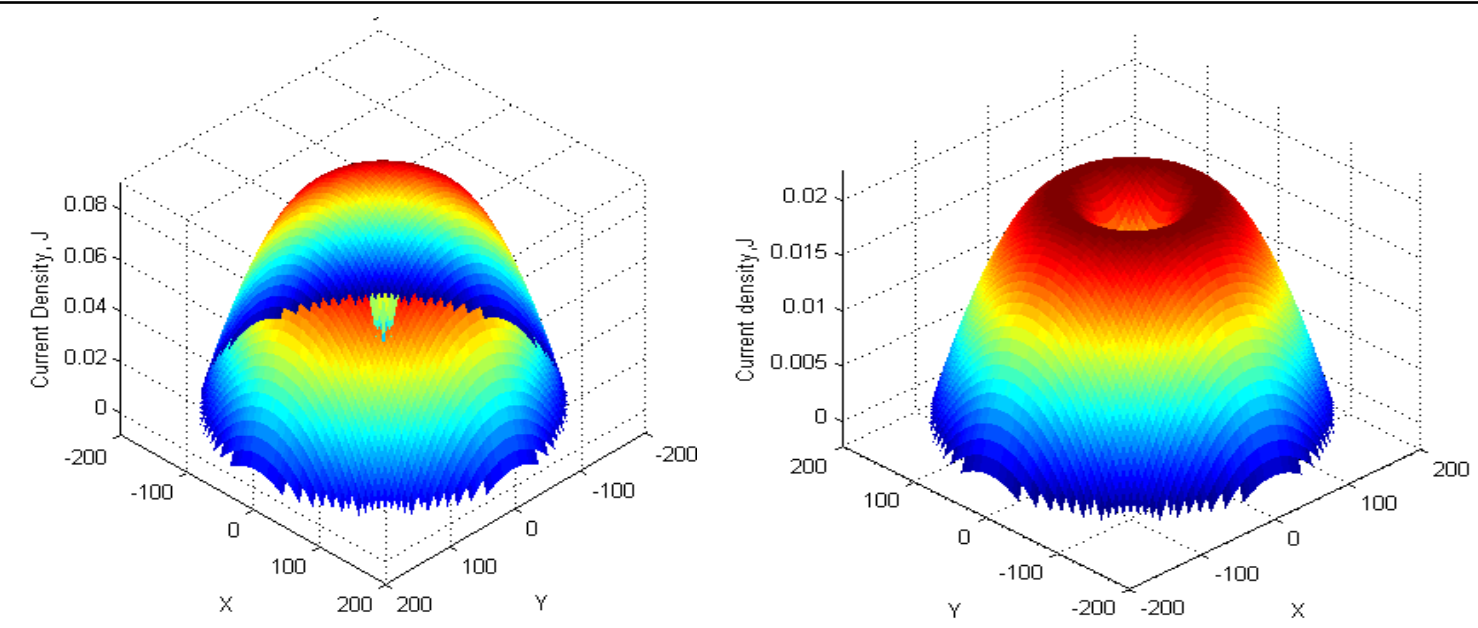

Figure 3. Bottom view (left) and side view (right) of the variation of the current density with core radius $r$ for $\mathrm{NbN}(\varepsilon=3-5 \mathrm{~nm}$ and $\lambda=200-350 \mathrm{~nm})$ in GL theory.

\section{CLASSICAL PROBABILITY DENSITY}

Cooper pairs were treated classically as an ideal gas particle interacting weakly with each other satisfying Maxwell-velocity distribution. Number of molecules with velocity vectors terminating in a thin slice perpendicular to the $\mathrm{x}$ and $\mathrm{y}$ axis and at a distance from the origin in momentum space is

$$
d N_{p_{x} p_{y}}=\frac{N}{\pi}\left(\frac{1}{2 m k_{B} T}\right) \exp \left(-\frac{p_{x}^{2}+p_{y}^{2}}{2 m k_{B} T}\right) d p_{x} d p_{y}
$$

where $m$ is the mass of the cooper pair, $k_{B}$ is the Boltzmann constant, $T$ is the super conducting temperature and $N$ is the total number of cooper pairs within the slice. Cooper is a common property for every superconductor and only the superconducting temperature depend on the material. $\mathrm{Zn}$ metal was taken as the superconducting material and with Superconducting temperature $0.85 K$ [7]. The momentum probability variation of cooper pairs with respect to momentum presented in the Figure 5 show a Gaussian probability peak around zero momentum. 


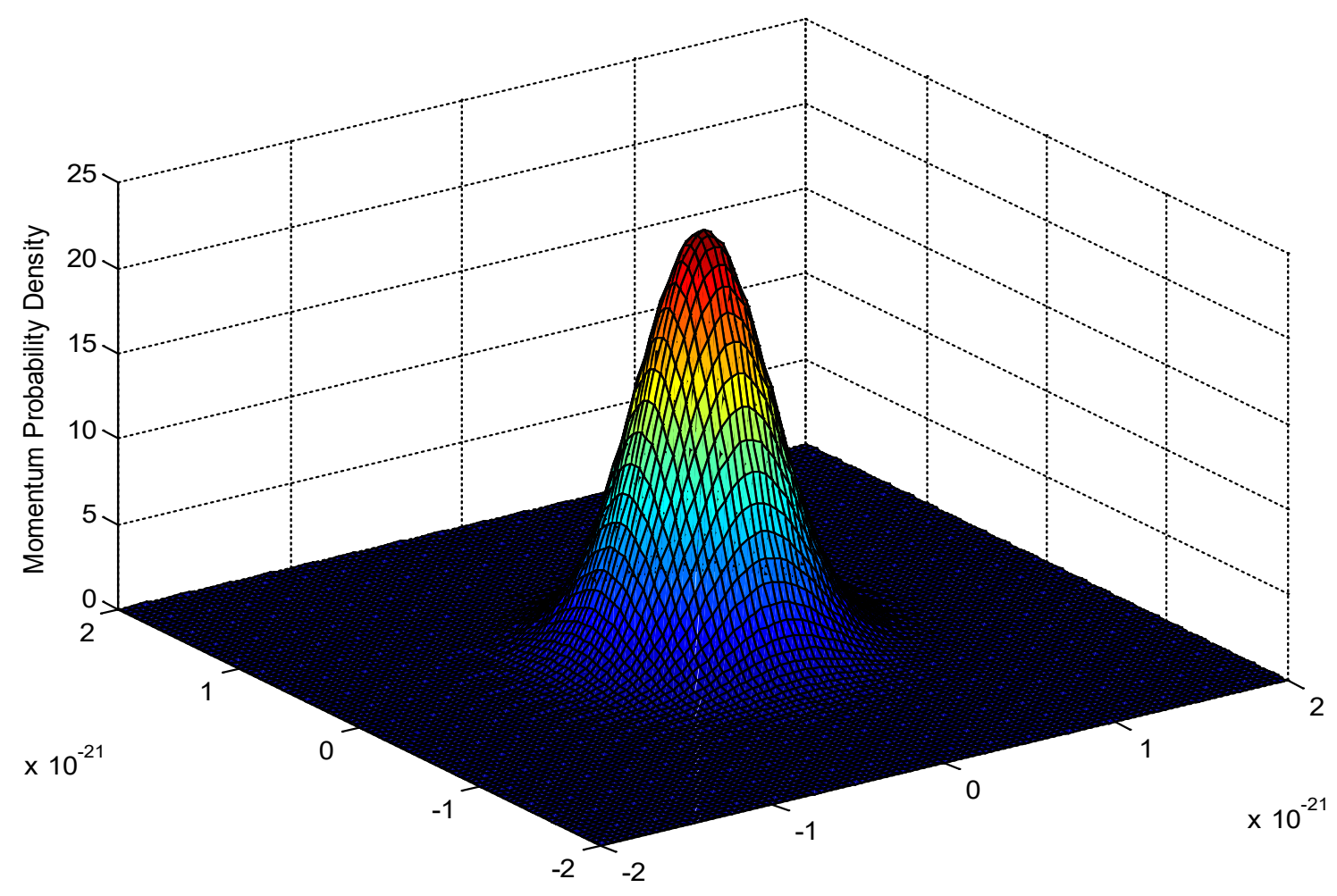

Fig. 5. Distribution of classical momentum probability density of cooper pairs in $\mathrm{Zn}$ with superconducting temperature $0.85 K$

\section{BCS THEORY}

One of the significant achievements of the BCS theory is the explanation that the cooper pairs must have a definitive quantum mechanical phase $\theta$ and consequently that the particle number $N$ is not fixed from the uncertainty principle. A Cooper is a single electron pair outside an occupied Fermi surface. The fermions forms a stable pair, bound state, no matter how weak is the attractive force. By constructing a many particles wave function in which all the electrons near the Fermi surface are paired up, that is a form of a coherent state, the energy gap was obtained by Schreifer. This energy gap $2 \Delta$ is the energy required to break up a pair into two free electrons. BCS theory gives the superconducting transition temperature $T_{\mathrm{c}}$ in terms of the electron-phonon coupling potential $V$ and the Debye cutoff energy $E_{\mathrm{D}}$. The electrons lie within $\pm k_{B} T$ of the Fermi energy and $h v_{D} \geq k_{B} T$ ( $v_{D}$ Debye frequency).

In the ground state, the spatial parts of the two wave functions should be identical. It is also expected that the electron wave components constituting the Cooper pair will have the wave vectors near the Fermi wave vector $k_{F}$ at the top of the Fermi sea. The size of the Cooper pair is inversely proportional to the effective bandwidth of the band of electron components contributing to the pairing. Within the BCS theory, this effective bandwidth also provides self-consistently the binding energy of the Cooper pair. When the distance $d$ between the two electrons is smaller than the Fermi wavelength $\lambda_{F} \approx 2 d$ the angular momentum of the Cooper pair can be assumed to be zero. Therefore electron pair move against each other along very close, parallel paths and that they are equally likely to do so in any direction. That is, the wave function should be spherically symmetric. The Schrodinger equation is then of the same form as that of the ground state electron of the hydrogen atom: 
$-\frac{\hbar^{2}}{2 \mu r^{2}} \frac{1}{R} \frac{d}{d r}\left[r^{2} \frac{d R}{d r}\right]+V(r) R=E R$

By using a new function $\psi(r)=r R(r)$ the equation 35 read

$-\frac{\hbar^{2}}{2 \mu} \frac{d^{2} \psi}{d r^{2}}+V(r) \psi=E \psi$

Under the quantum mechanical theory, the wave function of the cooper pairs can be obtained as

$\psi(r)=\sum_{\varepsilon=0}^{\hbar \omega_{D}} \frac{1}{(\varepsilon+\Delta)} \sin k r$

where $k$ is related to $\varepsilon$ by $\varepsilon=\frac{\hbar^{2} k^{2}}{m_{e}}-E_{F}, \quad \omega_{D}=\left(\frac{6 \pi^{2} N v^{3}}{V}\right), \Delta=\hbar \omega_{D} \exp \left(-2 \pi k_{F} / m_{e} l V_{0}\right)^{1 / 3}$

Changing the wave function to a new function $R(r)=\psi(r) / r$, the actual wave function read

$R(r)=\frac{1}{r} \sum_{\varepsilon=0}^{\hbar \omega_{D}} \frac{1}{(\varepsilon+\Delta)} \sin k r$

Since $r$ is the radial distance, the wave function can be converted into the Cartesian coordinate by taking $r=\sqrt{x^{2}+y^{2}}$. Then the actual wave function in coordinate space becomes

$R(x, y)=\frac{1}{\sqrt{x^{2}+y^{2}}} \sum_{\varepsilon=0}^{\hbar \omega_{D}} \frac{1}{(\varepsilon+\Delta)} \sin \left(k \sqrt{x^{2}+y^{2}}\right)$

To find out the momentum space wave function, it is Fourier transformed into the momentum space. If the new wave function is $\phi(p)$, then

$\phi(p)=\frac{1}{\sqrt{2 \pi \hbar}} \int_{0}^{\infty} e^{-i p \cdot r / \hbar} R(r) d r$

By substituting $r$ in terms of $x$ and $y$ the momentum space wave function can be derived in terms of Cartesian coordinates.

$\phi(p)=\frac{1}{\sqrt{2 \pi \hbar}} \iint_{0}^{\infty} e^{-i\left(p_{x} x+p_{y} y\right)} \frac{1}{\sqrt{x^{2}+y^{2}}} \sum_{\varepsilon=0}^{\hbar \omega_{D}} \frac{1}{(\varepsilon+\Delta)} \sin \left(k \sqrt{x^{2}+y^{2}}\right) d x d y$

The momentum probability density, 
$P_{n}(p)=\left|\frac{1}{\sqrt{2 \pi \hbar}} \iint_{0}^{\infty} e^{-i\left(p_{x} x+p_{y} y\right)} \frac{1}{\sqrt{x^{2}+y^{2}}} \sum_{0}^{\hbar \omega_{D}} \frac{1}{(\varepsilon+\Delta)} \sin \left(k \sqrt{x^{2}+y^{2}}\right) d x d y\right|^{2}$

Considering the Cooper pair as a boson particle with the spin zero, the quantum mechanical momentum probability density is shown in Figure 6 for $\mathrm{Zn}$ metal. Fermi energy of the superconducting material was taken as $9.46 \mathrm{eV}$, the velocity of sound inside the superconducting material $4320 \mathrm{~ms}^{-1}$ and the total number of free electron $N / V=132 \times 10^{27}$.

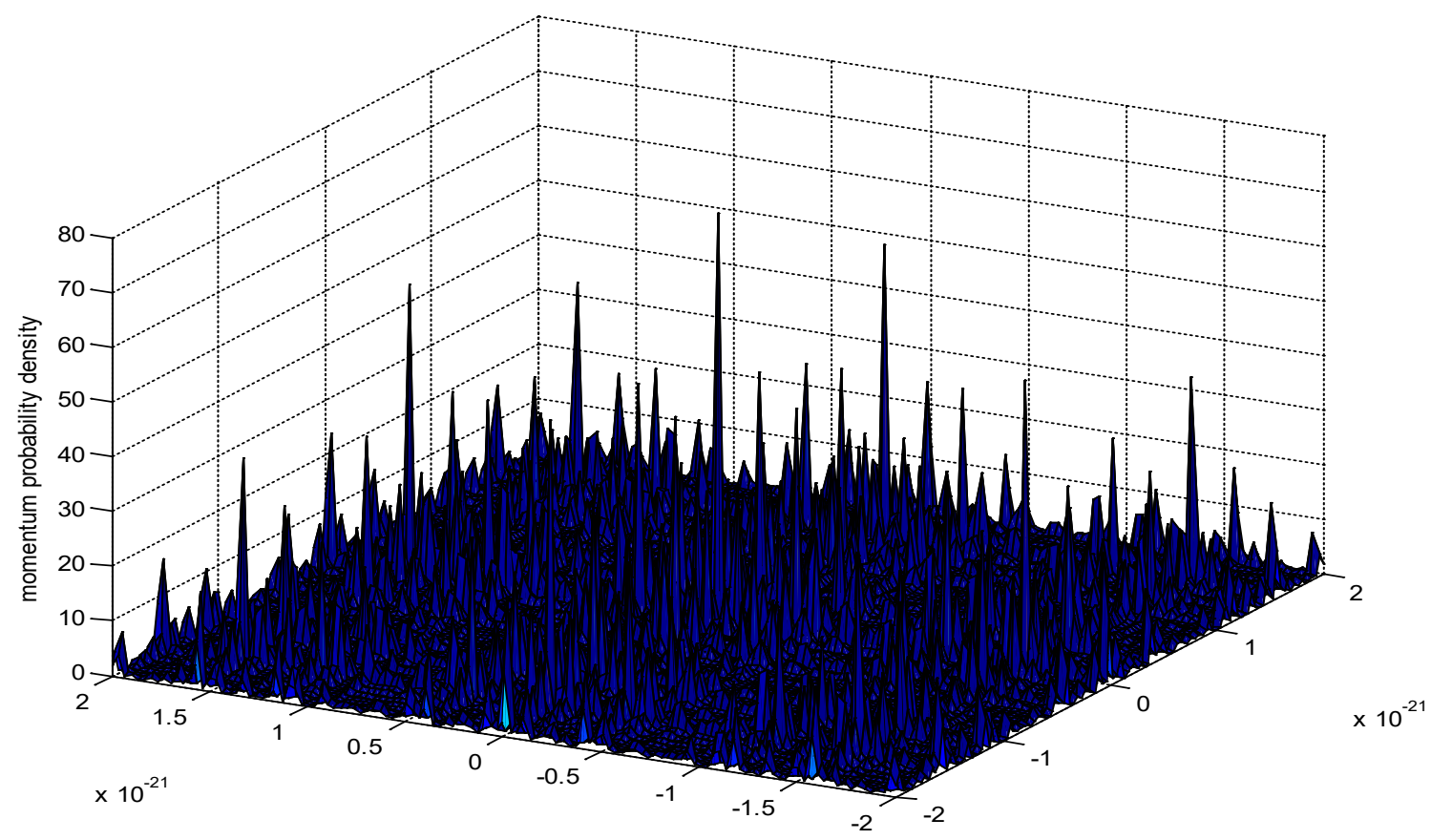

Figure 6. Momentum probability density distribution of Cooper pairs for $\mathrm{Zn}$.

Many peaks at different momentums can be observed in the graph. Both classical and quantum mechanical probability densities of Cooper pairs are presented in Figure 7. Light blue colour region represents the classical distribution of the momentum probability density of cooper pairs while dark Colour peaks represent the quantum mechanical momentum probability distribution. The classical mechanical probability density peaked at the zero momentum and the quantum mechanical probability density distribution peaks observed for many momenta, overlap at zero momentum. Classically, the cooper pairs are condensed into the region where their momentum approximately equal to the zero value and hence the cooper pairs have negligible velocity. But in quantum mechanics Cooper pairs are moving with a considerable velocity distribution which can be attributed to many momentum probability peaks. As the classical mechanical treatment shows a peak at near the zero momentum, the quantum mechanical momentum probability density gives the highest peak at the zero point. 


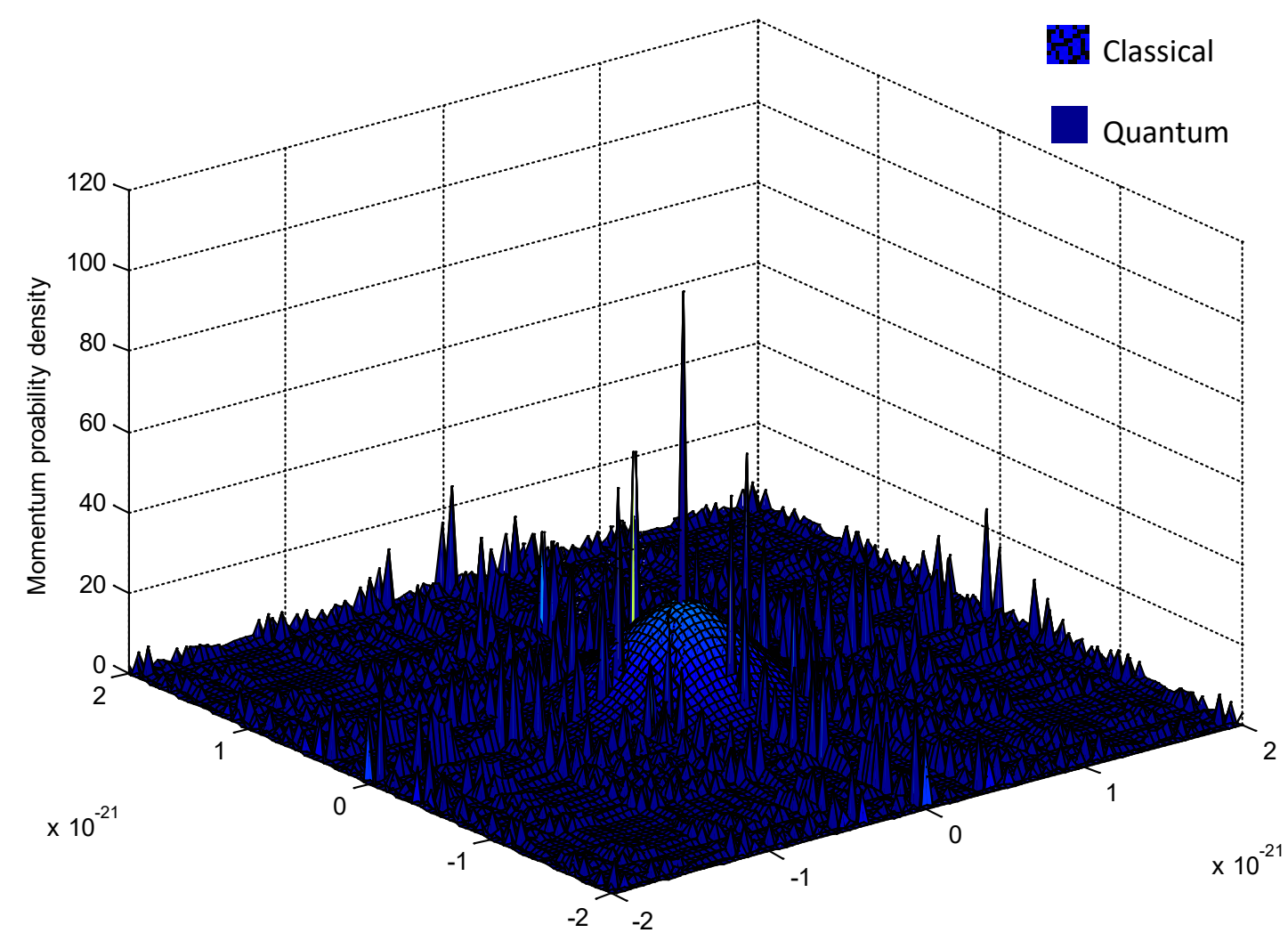

Figure 7. Classical (Maxwell distribution) and quantum mechanical (BCS theory) momentum probability density distribution of Cooper pairs in $\mathrm{Zn}$.

\section{CONCLUSION}

Under London method and GL method at classical limit, that is when there is no momentum, the same current density distributions were obtained for the superconducting material $\mathrm{NbN}$. When the cooper pair was considered as a complete classical particle with zero spin with Maxwell velocity distribution, the probability density was condensed into a small region near zero momentum. The most high momentum density of cooper pairs occurs at zero momentum as cooper pairs remain stationary inside a superconductor.

The classical momentum or position probability density model used for cooper pairs for $\mathrm{Zn}$ superconductor, gave results that can be accepted with the quantum model.

\section{References}

[1] O. H. Kamerlingh, Comm. Phys. Lab. Univ. Leiden 120b, 122b, 124c. (1911)

[2] W. Meissner, R. Ochsenfeld, Naturwissenschaften 21 (1933) 787.

[3] F. London, H. London, Proc. Roy. Soc. (London) A149 (866) (1935) 71-88.

[4] V. L. Ginzburg, L. D. Landau, Zh. Eksp. Teor. Fiz. 20 (1950) 1064.

[5] L. N. Cooper, Phys. Rev. 104 (1956)1189-1190. 
[6] J. Bardeen, L. N. Cooper, J. R. Schrieffer, Phys. Rev. 106 (1) (1957) 162-164.

[7] V. L Ginzburg, Chem. Phys. Chem. 5 (7) (2004) 930-945.

[8] A. M. Kadin, J. Supercond. \& Novel Mag. 20 (2007) 285-292.

( Received 15 October 2014; accepted 27 October 2014 ) 